\title{
Proteomic analysis of purified protein derivative of Mycobacterium tuberculosis
}

\author{
Thottethodi Subrahmanya Keshava Prasad ${ }^{1,2,3,4^{*}}$, Renu Verma ${ }^{1,4,5}$, Satish Kumar ${ }^{6}$, Raja Sekhar Nirujogi ${ }^{1,2}$, \\ Gajanan J Sathe ${ }^{1,3}$, Anil K Madugundu', Jyoti Sharma ${ }^{1,3}$, Vinuth N Puttamallesh', Anjali Ganjiwale, \\ Vithal P Myneedu ${ }^{7}$, Aditi Chatterjee ${ }^{1,3}$, Akhilesh Pandey $^{8^{*}}$, HC Harsha $^{1}$ and Jayasuryan Narayana ${ }^{5^{*}}$
}

\begin{abstract}
Background: Purified protein derivative (PPD) has been used for more than half a century as an antigen for the diagnosis of tuberculosis infection based on delayed type hypersensitivity. Although designated as "purified," in reality, the composition of PPD is highly complex and remains ill-defined. In this report, high resolution mass spectrometry was applied to understand the complexity of its constituent components. A comparative proteomic analysis of various PPD preparations and their functional characterization is likely to help in short-listing the relevant antigens required to prepare a less complex and more potent reagent for diagnostic purposes.

Results: Proteomic analysis of Connaught Tuberculin 68 (PPD-CT68), a tuberculin preparation generated from M. tuberculosis, was carried out in this study. PPD-CT68 is the protein component of a commercially available tuberculin preparation, Tubersol, which is used for tuberculin skin testing. Using a high resolution LTQ-Orbitrap Velos mass spectrometer, we identified 265 different proteins. The identified proteins were compared with those identified from PPD M. bovis, PPD M. avium and PPD-S2 from previous mass spectrometry-based studies. In all, 142 proteins were found to be shared between PPD-CT68 and PPD-S2 preparations. Out of the 354 proteins from M. tuberculosis-derived PPDs (i.e. proteins in either PPD-CT68 or PPD-S2), 37 proteins were found to be shared with M. avium PPD and 80 were shared with M. bovis PPD. Alignment of PPD-CT68 proteins with proteins encoded by 24 lung infecting bacteria revealed a number of similar proteins (206 bacterial proteins shared epitopes with 47 PPD-CT68 proteins), which could potentially be involved in causing cross-reactivity. The data have been deposited to the ProteomeXchange with identifier PXD000377.

Conclusions: Proteomic and bioinformatics analysis of different PPD preparations revealed commonly and differentially represented proteins. This information could help in delineating the relevant antigens represented in various PPDs, which could further lead to development of a lesser complex and better defined skin test antigen with a higher specificity and sensitivity.
\end{abstract}

Keywords: Biomarker, Broad spectrum antibiotics, Epitope, LC-MS/MS, Mantoux test

\section{Background}

Around 2 billion people in the world are infected with M. tuberculosis. According to WHO world TB (tuberculosis) control report 2011, in 2010 alone, 9 million new TB cases were reported and 1.45 million deaths occurred worldwide. Tuberculosis is the second most common

\footnotetext{
* Correspondence: keshav@ibioinformatics.org; pandey@jhmi.edu; jayasuryanmn@gmail.com

${ }^{1}$ Institute of Bioinformatics, International Tech Park, Whitefield, Bangalore 560 066, India

${ }^{2}$ Centre for Bioinformatics, School of Life Sciences, Pondicherry University, Puducherry 605 014, India

Full list of author information is available at the end of the article
}

infectious killer disease after HIV. One in five of the 1.8 million AIDS related deaths are estimated to be associated with TB. Tuberculin skin test (TST) is the standard test for the diagnosis of TB infection in the Western world [1]. American Thoracic Society and Center for Disease Control and Prevention recommend targeted TST for deciding the treatment regimen among groups associated with increased risk for progression of latent tuberculosis infection (LTBI) to active TB [2]. Vaccination is an important preventive measure to control community load of TB. An attenuated strain of $M$. bovis known as Bacillus Calmette-Guerin (BCG) is universally

\section{Biomed Central}


employed as a vaccine against TB. However, efficacy of BCG is controversial as it does not protect against adult forms of pulmonary tuberculosis [3,4]. Moreover, prior exposure of individual to environmental mycobacteria and organisms sharing antigenic epitopes results in recall of immune memory response to BCG administration [3]. After almost 12 decades of research, we still do not have a reliable diagnostic test for TB that can be used in primary health care centers with definitive results.

In 1890, Robert Koch introduced boiled, crude extract of tubercle bacilli in glycerin (referred as "old tuberculin") as a potential vaccine material against tuberculosis infection [5-7]. Although Koch's old tuberculin could not be used as therapy because of its toxicity, impurity and inadequate standardization; the concept of tuberculin was instrumental in laying the foundation of the modern TST [8]. TST, first introduced by Von Pirquet in 1909 [6] has been in use as a standard method for diagnosing TB infection almost over the last six decades $[8,9]$. It is based on measuring the extent of induration formed because of delayed type hypersensitivity reaction elicited by mycobacterial antigens present in PPD.

In addition to its role in detecting mycobacterial infection, TST has also been used as a standard tool to estimate the prevalence of LTBI [8]. The role of PPD in serodiagnosis of $\mathrm{TB}$, with sensitivity as high as $92 \%$, was reported in Warao and Creole populations [10]. Several studies reported the use of PPD in serodiagnosis of tuberculosis infection with high sensitivity [11,12]. PPD has also been used as a standard control in immunological assays [13]. It is reported that PPD improves the sensitivity of interferon gamma release assay (IGRA). IGRA uses early secretory antigenic target-6 (ESAT-6) and culture filtrate antigen EsxB (CFP10) antigens present in M. tuberculosis and M. bovis but not in BCG. This can enable differentiation of TB-infected and BCG vaccinated individuals [14,15]. However, Yassin et al. reported that sensitivity of IGRA can be compromised in children with severe malnutrition and HIV coinfection. Concomitant use of TST, IGRA and interferon gamma induced protein 10 (IP-10) in children staying in contact with smear-positive adults has shown higher number of children as positive [16]. In addition, IGRAs suffer from limitations including higher cost, variable sensitivity, poor reproducibility, limited interpretive criteria and unknown prognostic value [17]. Despite its important applications, PPD is not considered as a reliable material. This is due to high rates of false positive results, inability to distinguish between tuberculous and non-tuberculous mycobacteria or individuals vaccinated with BCG [18]. This can be attributed to immune response elicited by antigens from BCG or environmental bacteria sharing antigenic epitopes $[19,20]$. Earlier studies by Borsuk et al. identified molecular chaperone DnaK
(DnaK), molecular chaperone GroEL (GroEL2), elongation factor 2 (EF-Tu), cell surface lipoprotein Mpt83 (Mpt83) and acyl carrier protein as abundant proteins common to M. bovis and M. avium PPDs [21]. Moreover, discrepancy of results has been observed between different PPD preparations [22,23]. Currently available PPD preparations used on human subjects include PPD-S2 [6], PPD-RT23 [24], PPD IC-65 [9,13] and PPD-CT68 [25].

Knowledge about the constituents of PPD could allow the researchers to effectively work on PPD associated diagnostic complications. Earlier studies employed gel electrophoresis to identify constituents of PPD [26]. Kuwabara and Tsumita in 1974 first attempted to identify and characterize the components of PPD [27]. An analysis which employed gel electrophoresis for characterization of PPD antigens in whole cell lysate of $M$. bovis BCG resulted in four protein bands corresponding to PPD [28]. Kitaura et al. could distinctly identify only two ribosomal proteins L7 and L12 in M. tuberculosis and M. bovis PPDs in gel electrophoresis [29]. With the advent of high resolution mass spectrometry, it is now possible to identify proteins from complex peptide mixtures. Borsuk et al. identified 171 proteins in an LC-MS/MS analysis of Brazilian and UK bovine and avium PPDs [19]. Cho et al. recently identified 240 proteins in PPD-S2 [26]. PPD-CT68, which is another standard reagent used for TST, has not been analyzed thus far. In the present report, we have analyzed and described the proteome profile of PPD-CT68 using high resolution mass spectrometry and compared it with that of other PPDs derived from M. tuberculosis, M. avium and M. Bovis. PPD-CT68 examined here was developed by Landi in 1963 from "Johnston" strain of M. tuberculosis var. hominis [30].

\section{Results and discussion}

\section{Identification of proteins present in PPD-CT68 from} Mycobacterium tuberculosis

We carried out a proteomic profiling of PPD-CT68 prepared from $M$. tuberculosis culture in a protein-free medium using high resolution Fourier transform mass spectrometry. Mass spectrometry-derived data was searched using Sequest algorithm embedded in the Proteome Discoverer software against a protein database of M. tuberculosis from NCBI RefSeq. Search of 5,205 MS/ MS spectra resulted in 1,146 peptide-spectrum matches, which corresponded to 695 unique peptides. The list of peptides identified in this study is provided in Additional file 1: Table S1. Representative MS/MS spectra are provided in Figure 1. Based on these 695 unique peptides, we identified 265 proteins (Additional file 2: Table S2) of M. tuberculosis in PPD-CT68.

Cho and colleagues [26] recently reported the identification of 240 proteins from PPD-S2, which is the standard for TST, as recommended by U.S. Food and Drug 
A. HTIFGEVIDAesQR

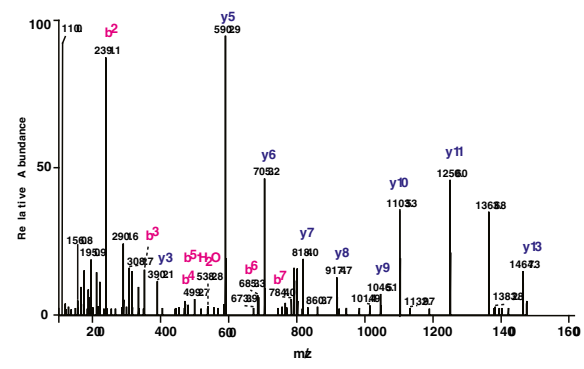

C.

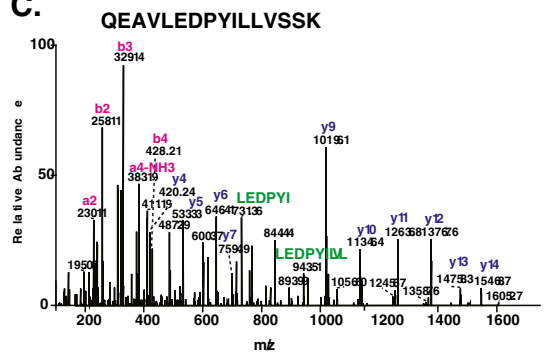

B. SPAGAWQYTAK

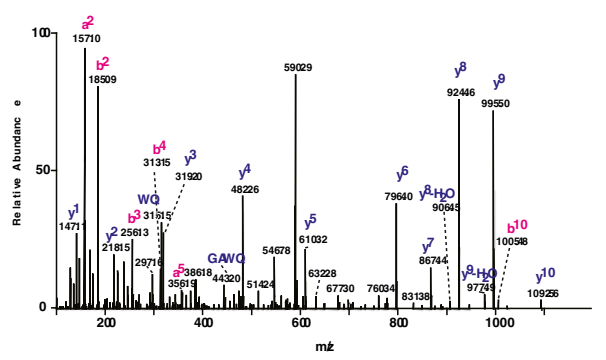

D. VDIAIVGLSTR

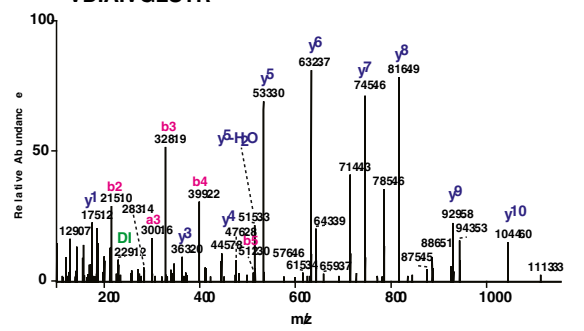

Figure $1 \mathrm{MS} / \mathrm{MS}$ spectra of peptides from some of the $M$. tuberculosis PPD-CT68 proteins identified in this study (A) iron-regulated peptidyl-prolyl cis-trans isomerase A (B) catalase-peroxidase peroxynitritase T KatG (C) molecular chaperone GroEL (D) polyketide synthase.

Administration (FDA). Out of 240 proteins listed, 231 are non-redundant. We compared proteomic results obtained in our study with proteins identified from PPD-S2 (Figure 2A). Out of the 265 proteins identified from PPDCT68, 142 proteins were shared with PPD-S2, whereas, 123 and 89 proteins were exclusively identified from PPDCT68 and PPD-S2, respectively. Altogether, 354 proteins of $M$. tuberculosis PPD have been identified.

For further understanding of protein profiles of various PPD preparations, we compared the PPD derived from M. tuberculosis (PPD-CT68 and PPD-S2) with PPDs of $M$. bovis and M. avium [19] (Figure 2B). Out of 354 proteins from $M$. tuberculosis PPDs, 37 proteins were found common with $M$. avium PPD and 80 were common with $M$. bovis PPD. We also found that 18 proteins were common in PPDs obtained from M. tuberculosis, M. bovis and M. avium. When compared to PPDs from M. tuberculosis, 35 and 19 proteins were exclusively found in $M$. avium and $M$. bovis PPDs respectively. It is also interesting to note that 255 proteins were exclusively identified in PPDs from M. tuberculosis.

Functional analysis of proteins common among all PPDs We carried out functional classification of proteins identified in M. tuberculosis (PPD-CT68 and PPD-S2), which are also present in $M$. avium and $M$. bovis. Most of the proteins were implicated in causing infection and protecting the pathogen against various metabolic stresses. Five of eighteen proteins- secreted antigen 85A (FbpA), thiol peroxidase $(\mathrm{Tpx})$, bacterioferritin (BfrA), thioredoxin
(TrxC) and lipoprotein LprG (LprG) - offer protection against oxidative and nitrosative stress. On the other hand, co-chaperonin GroES (GroES), DnaK, serine protease PepA (PepA), alanine and proline rich secreted protein Apa (Apa) and hypothetical protein Mpt64 are involved in causing infection. Detailed functional classification of each protein is given in the Table 1.

\section{Bioinformatics analysis of PPD-CT68 proteins showing homology to lung infecting bacteria}

Raman et al. performed a comprehensive analysis on M. tuberculosis genes homologous to 228 different pathogenic bacteria [50]. We further analyzed 265 proteins represented in PPD-CT68 against the proteins encoded by 24 lung infecting bacteria selected from the list of 228 pathogens. Protein BLAST was performed to locate regions of PPD-CT68 proteins sharing 10 or more identical amino acid long regions with bacterial proteins. In all, 3,446 peptides from 24 pathogens corresponding to 1,048 proteins showed 10 or more amino acid identity with 117 proteins from PPD-CT68 (Additional file 3: Table S3a and S3b). Since, a peptide of 20 or more amino acid residues can be a potential epitope, we further shortlisted the proteins reflecting identity in a continuous stretch of 20 or more amino acids (Additional file 3: Table S3c). Two hundred and six out of 1,048 bacterial proteins showed identity with 47 PPD-CT68 proteins. (Figure 3A). Functional analysis of 47 mycobacterial proteins sharing identical regions revealed that $41 \%$ proteins are associated with intermediary metabolism and respiration, 34\% with information 


\section{A}

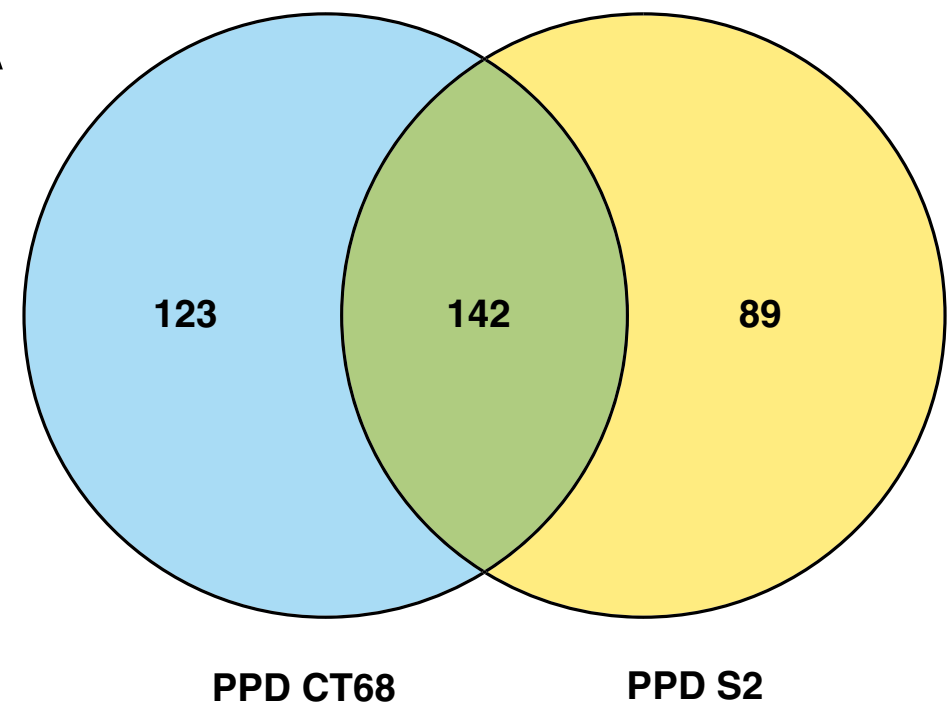

PPDs from M. tuberculosis

B

M. tuberculosis PPD

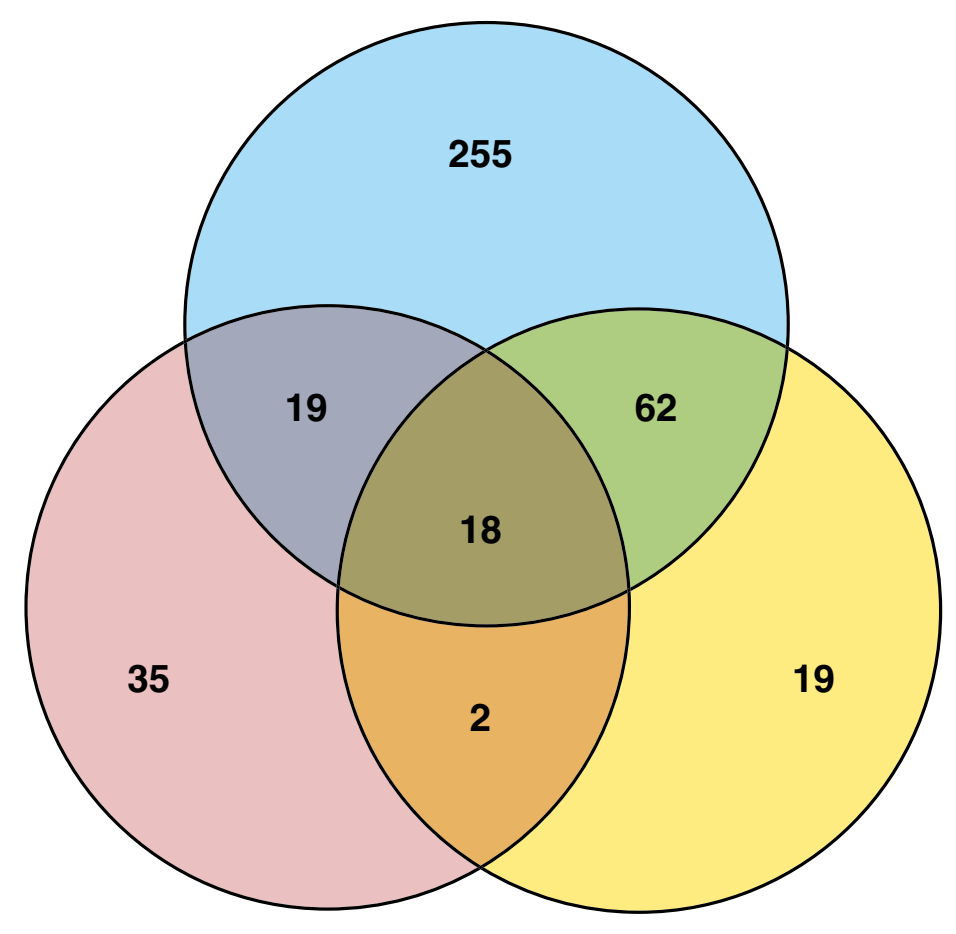

M. avium PPD

M. bovis PPD

Figure 2 Overlap of proteins identified among PPD-CT68 and PPD-S2 (A) Proteins identified among M. tuberculosis PPD, M. avium PPD and $M$. bovis PPD (B).

pathways, 9\% with virulence and detoxification, $4 \%$ with cell wall and cell processes, $4 \%$ with lipid metabolism and $4 \%$ with regulatory proteins (Figure $3 \mathrm{~B}$ ).
To further study the role of these 47 proteins in DTH and serodiagnosis, we compared our data with immunoproteome of $M$. tuberculosis reported by Velayudhan et al., which 
Table 1 Functional classification of proteins common in all PPDs

\begin{tabular}{|c|c|c|c|c|}
\hline & $\begin{array}{l}\text { Gene } \\
\text { symbol }\end{array}$ & Description & Function & Reference \\
\hline 1 & AcpP & Acyl carrier protein & It shuttles the intermediates between the enzymes of type II fatty acid synthase system & [31] \\
\hline 2 & FbpA & Secreted antigen 85-A & $\begin{array}{l}\text { It participates in cell wall biosynthesis, and interacts with the host } \\
\text { macrophage as fibronectin-binding protein. It is also involved in } \\
\text { establishment and maintenance of a persistent tuberculosis infection }\end{array}$ & {$[32,33]$} \\
\hline 3 & GroES & Co-chaperonin & It is a dominant immunogenic protein & [34] \\
\hline 4 & Dnak & Molecular chaperone & $\begin{array}{l}\text { It is highly antigenic and act as co-repressors for heat shock protein } \\
\text { transcriptional repressor (hspR) }\end{array}$ & [35] \\
\hline 5 & Tpx & Thiol peroxidase & It protects $M$. tuberculosis against oxidative and nitrosative stress & [36] \\
\hline 6 & RpiL & 50 S ribosomal protein L7/L12 & It is involved in interaction with translation factors & {$[37]$} \\
\hline 7 & BfrA & Bacterioferritin & $\begin{array}{l}\text { It is an intracellular iron storage protein, which protects Mycobacterium } \\
\text { from oxidative stress mediated by excess iron }\end{array}$ & [38] \\
\hline 8 & SahH & S-adenosyl-L-homocysteine hydrolase & $\begin{array}{l}\text { It is a ubiquitous enzyme that plays a central role in methylation-based processes by } \\
\text { maintaining the intracellular balance between S-adenosylhomocysteine (SAH) } \\
\text { and S-adenosylmethionine }\end{array}$ & [39] \\
\hline 9 & TrxC & Thioredoxin & $\begin{array}{l}\text { It is involved in redox homeostasis and uses it to protect the pathoen against } \\
\text { the oxidative intermediates generated by the macrophages }\end{array}$ & [40] \\
\hline 10 & FixB & $\begin{array}{l}\text { Electron transfer flavoprotein subunit } \\
\text { alpha }\end{array}$ & $\begin{array}{l}\text { It is electron transfer flavoprotein subunit alpha, in some bacteria it functions } \\
\text { as nitrogen fixing agent but its function in } M \text {.tuberculosis is not clear }\end{array}$ & [41] \\
\hline 11 & PepA & Serine protease & $\begin{array}{l}\text { It is a serine protease associated with cell membrane, which stimulates peripheral blood } \\
\text { mononuclear cells from healthy PPD donors to proliferate and secrete gamma interferon }\end{array}$ & [42] \\
\hline 12 & Wag31 & Hypothetical protein & $\begin{array}{l}\text { It is a cell division initiation protein involved in regulation of genes } \\
\text { including virulence factors and antigens }\end{array}$ & [43] \\
\hline 13 & Mpt64 & Hypothetical protein & $\begin{array}{l}\text { It is an immunogenic protein which elicits delayed type hypersensitivity skin } \\
\text { response }\end{array}$ & [44] \\
\hline 14 & Apa & $\begin{array}{l}\text { Alanine and proline rich secreted } \\
\text { protein }\end{array}$ & $\begin{array}{l}\text { It is a cell surface glycoprotein which binds to host lectins and cheat } \\
\text { the innate immune system }\end{array}$ & [45] \\
\hline 15 & LprG & Lipoprotein LprG & $\begin{array}{l}\text { It plays a role in } M \text {. tuberculosis infection by inducing increased } \\
\text { suppression of the immune response due to elevated nitric oxide production }\end{array}$ & [46] \\
\hline 16 & Rv1893 & Hypothetical protein & Function unknown & {$[47]$} \\
\hline 17 & Rv1855c & Oxidoreductase & Probable monooxygenase & [48] \\
\hline 18 & Gap & $\begin{array}{l}\text { Glyceraldehyde-3-phosphate } \\
\text { dehydrogenase }\end{array}$ & It has glyceraldehyde-3-phosphate dehydrogenase activity & [49] \\
\hline
\end{tabular}

included 484 mycobacterial proteins recognized by human sera collected from worldwide TB suspects [51]. Thirteen proteins - DNA-directed RNA polymerase subunit beta; DNA-directed RNA polymerase subunit alpha; GroEL; $30 S$ ribosomal protein S1; fumarate hydratase; elongation factor G; DnaK; aconitate hydratase; isocitrate dehydrogenase; S-adenosyl-L-homocysteine hydrolase; malate synthase G; D-3-phosphoglycerate dehydrogenase; and enoyl-CoA hydratase - were recognized by antibodies in serum. Out of these 13 proteins, only seven (isocitrate dehydrogenase; malate synthase G; succinyl-CoA synthetase subunit alpha; malate dehydrogenase; succinyl-CoA synthetase subunit beta; aconitate hydratase; and type II citrate synthase) were listed in immunoproteome and were identified in our mass spectrometry analysis of PPD-CT68 [51]. These proteins are associated with a host immune response in cases with active tuberculosis.

\section{PPD proteins as candidate biomarkers}

Available knowledge of the $M$. tuberculosis genes provides us the advantage to express and synthesize recombinant purified antigens, which gives us the opportunity to test new biomarkers for TB infection. These antigens can be used to detect the antibodies in the serum and have a potential to improve diagnosis. Several studies have explored the use of the recombinantly expressed antigens and evaluated their immunodiagnostic potential. An updated systematic review on the diagnostic accuracy of commercial serological tests for pulmonary and extra pulmonary tuberculosis for relevant studies was updated in May 2010 and WHO has performed a bivariate meta-analysis that jointly modeled both test sensitivity and specificity (http://www.who.int/tb/laboratory/ policy_statements/en/index.html). It has been concluded that the commercially available serological tests provide inconsistent and imprecise findings and the sensitivity and 


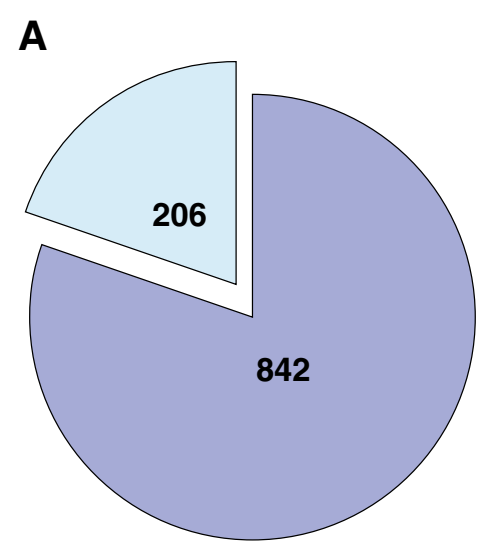

B

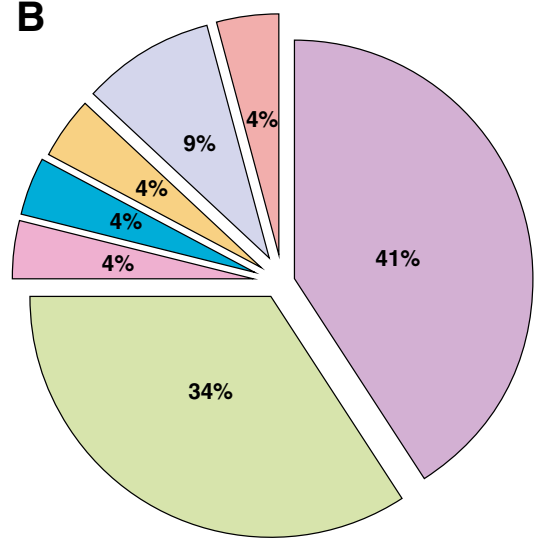

Proteins with >=20 amino acid identity

Proteins with $<20$ amino acid identity
Lipid metabolism

Virulence, detoxificaiton and adaptation

Regulatory proteins

Cell wall and cell processes

Conserved hypotheticals

Information pathways

Intermediary metabolism and respiration

Figure 3 Pie chart representing lung infecting bacterial proteins with $<20$ amino acid long peptides and $\geq \mathbf{2 0}$ amino acid long peptides identical to PPD-CT68 proteins (A). Functional classification of 47 PPD-CT68 proteins showing identity of 20 or more amino acids in 24 lung infection associated bacterial proteins (B).

specificity of the tests were highly variable. Our approach presented in this study, has not only identified a large number of proteins unique to $M$. tuberculosis, but in parallel provided the information on the coverage. The higher the coverage, higher is the abundance of the protein in the PPD sample analyzed. Our results are correlating with earlier publications and many of the proteins identified in the PPD of M. tuberculosis, have already been analyzed for their potential as a diagnostic markers. Some of the hits we have found are GroES [52,53], GroEL [54,55], protein EsxB (EsxB) [56] heat shock protein HspX (HspX) [57], hypothetical protein (TB15.3), hypothetical protien (TB16.3) [58], 50S ribosomal protein L7/L12 (RplL) [59], hypothetical protein (EsxA) [60], immunogenic protein Mpt63 (Mpt63) [61], Mpt64 [62], ESAT-6 like protein EsxJ (EsxJ), and ESAT-6 like protein EsxO (EsxO) [63]. Based on these observations, many more M. tuberculosis PPD can be analyzed and the abundant antigens be evaluated for their potential as diagnostics biomarkers.

\section{Clinical applications of the study}

Based on our findings, out of 265 proteins identified in PPD-CT68, 142 proteins were found common between PPD-CT68 and PPD-S2. The common proteins can further be evaluated for their potential as skin test antigens. Proteins identified in our analysis, which are absent in M. avium and $M$. bovis and do not show any significant identity with proteins from lung infecting bacteria can be shortlisted for developing various immunological assays to identify $M$. tuberculosis, on the basis of their seroreactivity and abundance. For example, Rv2346, Rv0379 and Rv1388 were found to be absent in PPD M. bovis and PPD M. avium and possessed least identity with proteins from lung infecting pathogens. As discussed earlier, one of the major issues with the use of PPD as a skin test antigen is false positive results for the individuals with BCG vaccination. Use of antigens absent in $M$. bovis may help overcoming that. Global profiling of antigens in PPD may help to identify $M$. tuberculosis-specific antigens, which are not present in BCG. These antigens will be useful in 
differentiating infected from vaccinated individuals. PPD can be prepared from M. tuberculosis, M. avium and $M$. bovis, so if we can use antigen specific to every strain to elicit the test response we can diagnose the species of Mycobacteria with a simple skin test. Subset of antigens that is mainly responsible for activating the immune response can be used in adjunction with BCG or for booster doses to enhance immune response. By knowing the antigens involved in the test response, we can use minimal essential amount of PPD for TST. Use of specific antigens in TST will make it more specific and will reduce the false positive results due to antigen cross reaction.

\section{Conclusions}

Despite the identification of almost a dozen antigens for developing next generation PPD, it is challenging to replace the classical PPD preparation. ESAT-6, Mpt64, recombinant antigen (DPPD), CFP10, recombinant truncated $38 \mathrm{kDa}$ protein (TPA38), DnaK, GroEL, RplL are currently under evaluation as next generation PPD candidates $[9,29,62,64-72]$. PPD is a crude extract obtained after several steps of filtration, purification, and precipitation with trichloroacetic acid [30]. Enough knowledge on PPD composition and contribution of individual antigens in TST would give a better insight to understand the molecular mechanism behind it and will also allow the researchers to select a combination of proteins specific to M. tuberculosis. Our analysis further revealed mycobacterial proteins in PPD-CT68 sharing identical amino acid sequence with lung infecting bacteria. Detailed epitopic analysis of these proteins may help the researchers to understand the mechanism behind cross reactivity in TST.

Mass Spectrometry is an efficient tool for proteomic analysis due to its high mass accuracy, sensitivity and ability to deal with complex sample mixtures. Here, in this study we used a high resolution Fourier transform mass spectrometer for LC-MS/MS analysis of PPDCT68. Many of the proteins identified in the PPD of M. tuberculosis, have already been analyzed for their potential as diagnostic markers. The complete protein profile of PPD-CT68 uncovered from this study can be used to analyze immune response and antibody production pattern of body against different PPD antigens.

\section{Data availability}

The mass spectrometry proteomics data have been deposited to the ProteomeXchange Consortium (http:// proteomecentral.proteomexchange.org) via the PRIDE partner repository [73] with the dataset identifier PXD000377.

\section{Methods}

\section{Trypsin digestion}

PPD-CT68 (100 $\mu \mathrm{g}$ protein) was subjected to in-solution digestion using trypsin. Reduction and alkylation were carried out using $5 \mathrm{mM}$ dithiothreitol $\left(60^{\circ} \mathrm{C}\right.$ for $\left.45 \mathrm{~min}\right)$ and $20 \mathrm{mM}$ iodoacetamide (RT for $10 \mathrm{~min}$ ), respectively. Overnight digestion was carried out using trypsin with an enzyme: substrate ratio of $1: 20$ at $37^{\circ} \mathrm{C}$ [74]. The digest was then purified using sep-pak C18 columns (WAT051910, Waters Corporation, MA) [75] and the eluates were lyophilized at $-52^{\circ} \mathrm{C}$ (Operon, Gyeonggi-do, Korea) and stored at $-80^{\circ} \mathrm{C}$ until LC-MS/MS analysis.

\section{Mass spectrometry}

We have carried out the LC-MS/MS analysis on an LTQOrbitrap Velos ETD mass spectrometer (Thermo Scientific, Bremen, Germany) interfaced with an Agilent 1100 HPLC system (Agilent Technologies, Santa Clara, CA). Trypsin digested PPD peptides were analyzed on a reversed phase liquid chromatography. The RP-LC system equipped with a pre-column $\left(2 \mathrm{~cm}, 5 \mu-100 \mathrm{~A}^{\circ}\right)$, analytical column $\left(10 \mathrm{~cm}, 5 \mu-100 \mathrm{~A}^{\circ}\right)$ made with magic AQ $\mathrm{C}_{18}$ material (PM5/61100/00; Bruker-Michrom Inc., Auburn, CA) packed in-house. Further, the peptides were sprayed using an electro-spray emitter tip $8 \mu$ (New Objective, Woburn, MA) fixed to a nanospray ionization source. The peptides were loaded on the pre-column using $97 \%$ solvent A $(0.1 \%$ formic acid (aq) and resolved on the analytical column using a gradient of $10-30 \%$ solvent $\mathrm{B}$ (90\% acetonitrile, $0.1 \%$ formic acid) for $60 \mathrm{~min}$ at a constant flow rate of $0.35 \mu \mathrm{l} / \mathrm{min}$. The spray voltage and heated capillary temperature were set to $2.0 \mathrm{kV}$ and $220^{\circ} \mathrm{C}$, respectively. The data acquisition was performed in a data dependent manner. From each MS survey scan, 10 most intense precursor ions were selected for fragmentation. MS and MS/MS scans were acquired in an Orbitrap mass analyzer and the peptides were fragmented by higher energy collision dissociation with normalized collision energy of $39 \%$. MS scans were acquired at a resolution of 60,000 at $400 \mathrm{~m} / \mathrm{z}$, while $\mathrm{MS} / \mathrm{MS}$ scans were acquired at a resolution of 15,000 . The automatic gain control for full FT MS was set to 0.5 million ions and for FT MS/MS was set to 0.1 million ions with maximum time of accumulation of $750 \mathrm{~ms}$ and $100 \mathrm{~ms}$, respectively. The raw data obtained was submitted to ProteomeXchange Consortium (http://proteomecentral.proteomexchange.org).

\section{Data analysis}

We searched the MS/MS data using Sequest search algorithm on Proteome Discoverer (version 1.3.0.339, Thermo Scientific, Bremen, Germany), against protein database of M. tuberculosis $\mathrm{H} 37 \mathrm{Rv}$ strain downloaded from NCBI RefSeq (updated December 29, 2011). The search parameters are: a) precursor mass range between 350 to $8000 \mathrm{Da}$; b) minimum peak count was set to 5 ; c) signal to noise threshold set to 1.5 ; d) trypsin was used as a proteolytic enzyme allowing up to one missed cleavage; e) precursor mass tolerance of $20 \mathrm{ppm}$ and fragment tolerance 
of $0.1 \mathrm{Da}$; f) oxidation of methionine as variable modification and carbamidomethylation of cysteine as fixed modification; and g) $1 \%$ false discovery rate (FDR).

\section{Additional files}

Additional file 1: Table $\mathbf{S 1}$. List of peptides identified from M. tuberculosis
$\operatorname{PPD}(C T 68)$

Additional file 2: Table S2. List of proteins identified from $M$. tuberculosis PPD (CT68).

Additional file 3: Table S3a. List of Mycobacterium tuberculosis PPD-CT68 peptides with 10 or more amino acid identity with 24 lung infecting bacteria. Table S3b. List of Mycobacterium tuberculosis proteins corresponding to the peptides with 10 or more amino acid identity with 24 lung infecting bacteria. Table S3c. List of

Mycobacterium tuberculosis proteins with sequence identity of $\geq 20$ amino acid identity with 24 lung infecting bacteria.

\section{Abbreviations}

AcpP: Acyl carrier protein; Apa: Alanine and proline rich protein; BCG: Bacillus Calmette-Guerin; BfrA: Bacterioferritin; BLAST: Basic Local Alignment Search Tool; CFP10: Culture filtrate antigen EsxB; EF-Tu: Elongation factor 2; EsxJ: ESAT-6 like protein EsxJ; EsxO: ESAT-6 like protein EsxO; ESAT-6: Early secretory antigenic target-6; FbpA: Secreted antigen 85A; GroES: Cochaperonin GroES; HspX: Heat shock protein HspX; IGRA: Interferon gamma release assay; IP-10: Interferon gamma induced protein 10; LC-MS/MS: Liquid chromatography-mass spectrometry; LprG: Lipoprotein LprG; LTBI: Latent tuberculosis infection; Mpt83: Cell surface lipoprotein Mpt83; PepA: Serine protease PepA; PPD-CT68: Purified protein derivative-Connaught tuberculin 68; RpiL: 50S ribosomal protein L7/L12; SahH: S-adenosylhomocysteine hydrolase; Tpx: Thiol peroxidase; TrxC: Thioredoxin; TPA38: Truncated 38kDa protein; TST: Tuberculin skin test.

\section{Competing interests}

The authors declare that they have no competing interests.

\section{Authors' contribution}

JN, TSKP and AP conceived the study and designed the experiments; SK, NRS, GJS and HCH carried out the experiments; TSKP, HCH, RV, AKM and JS analyzed the data; TSKP, RV and VNP wrote the manuscript; JN, AG, AC and VPM provided critical comments. All authors read and approved the final manuscript.

\section{Acknowledgements}

Thottethodi Subrahmanya Keshava Prasad is the recipient of the DST-IDP research grant "Development of epitope based diagnostic gadget for detection of Mycobacterium tuberculosis in the Indian population" from the Department of Science Technology, Government of India. H.C. Harsha is a Wellcome Trust/DBT India Alliance Early Career Fellow. Renu Verma is a recipient of Junior Research Fellowship from University Grants Commission (UGC), Government of India. Gajanan J. Sathe is a recipient of a Junior Research Fellowship from the Council of Scientific and Industrial Research (CSIR), New Delhi, India. Anil K. Madugundu is a recipient of Junior Research Fellowship from Department of BiotechnologyBioinformatics National Certification (DBT-BINC). Jyoti Sharma is a recipient of a Senior Research Fellowship from the CSIR, New Delhi, India. This study was supported in part by an NIH roadmap grant for Technology Centers of Networks and Pathways (U54RR020839). The data deposition to the ProteomeXchange Consortium was supported by PRIDE Team, EBI.

\section{Author details}

${ }^{1}$ Institute of Bioinformatics, International Tech Park, Whitefield, Bangalore 560 066, India. ${ }^{2}$ Centre for Bioinformatics, School of Life Sciences, Pondicherry University, Puducherry 605 014, India. ${ }^{3}$ Manipal University, Madhav Nagar, Manipal 576 104, India. ${ }^{4}$ School of Biotechnology, KIIT University, Bhubaneswar, Odisha-751024, India. ${ }^{5}$ Microtest Innovations Pvt. Ltd., International Tech Park, Whitefield, Bangalore 560 066, India. ${ }^{6}$ Armed Forces Medical College, Pune 411 040, India. ${ }^{7}$ Department of Microbiology, LRS Institute of TB and RD, New Delhi 110 030, India. ${ }^{8}$ Johns Hopkins University School of Medicine, Baltimore, MD 21205, USA.
Received: 28 March 2013 Accepted: 16 July 2013

Published: 19 July 2013

\section{References}

1. Pineiro $R$, et al: Tuberculin skin test in bacille Calmette-Guerin-vaccinated children: how should we interpret the results? Eur J Pediatr 2012, 171(11):1625-32.

2. Targeted tuberculin testing and treatment of latent tuberculosis infection. American Thoracic Society. MMWR Recomm Rep 2000, 49(RR-6):1-51.

3. Brandt $L$, et al: Failure of the Mycobacterium bovis $B C G$ vaccine: some species of environmental mycobacteria block multiplication of BCG and induction of protective immunity to tuberculosis. Infect Immun 2002, 70(2):672-8.

4. Fine PE: Variation in protection by BCG: implications of and for heterologous immunity. Lancet 1995, 346(8986):1339-45.

5. Shingadia D, Novelli V: The tuberculin skin test: a hundred, not out? Arch Dis Child 2008, 93(3):189-90.

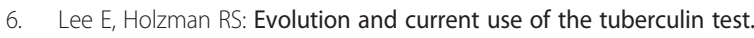
Clin Infect Dis 2002, 34(3):365-70.

7. Burke DS: Of postulates and peccadilloes: Robert Koch and vaccine (tuberculin) therapy for tuberculosis. Vaccine 1993, 11(8):795-804.

8. Al-Absi A, et al: The decline of tuberculosis in Yemen: evaluation based on two nationwide tuberculin surveys. Int J Tuberc Lung Dis 2009, 13(9):1100-5.

9. Yang $H$, Kruh-Garcia NA, Dobos KM: Purified protein derivatives of tuberculin - past, present, and future. FEMS Immunol Med Microbiol 2012, 66(3):273-80.

10. Araujo $Z$, et al: Comparison of serological responses in two different populations with pulmonary tuberculosis. Mem Inst Oswaldo Cruz 2008, 103(7):661-7.

11. Amicosante $M$, et al: Sensitivity and specificity of a multi-antigen ELISA test for the serological diagnosis of tuberculosis. Int J Tuberc Lung Dis 1999, 3(8):736-40.

12. Hwang PH, Kim JS: PPD-specific IgG and lgG subclasses in the sera of pulmonary tuberculosis patients. J Korean Med Sci 1993, 8(1):1-9.

13. Stavri HR, et al: Prospective Comparison of Two Brands of Tuberculin Skin Tests and Quantiferon-TB Gold in-tube Assay Performances for Tuberculosis Infection in Hospitalized Children. Maedica (Buchar) 2010, 5(4):271-6.

14. Buddle BM, et al: Use of ESAT-6 in the interferon-gamma test for diagnosis of bovine tuberculosis following skin testing. Vet Microbiol 2001, 80(1):37-46.

15. Buddle BM, et al: Differentiation between Mycobacterium bovis BCGvaccinated and $\mathrm{M}$. bovis-infected cattle by using recombinant mycobacterial antigens. Clin Diagn Lab Immunol 1999, 6(1):1-5.

16. Yassin $M$, et al: Added value of TST, IGRAS and IP-10 to identify children with TB infection. Eur Respir J 2012, 41(3):644-648.

17. Herrera V, et al: Clinical application and limitations of interferon-gamma release assays for the diagnosis of latent tuberculosis infection. Clin Infect Dis 2011, 52(8):1031-7.

18. Huebner RE, Schein MF, Bass JB Jr: The tuberculin skin test. Clin Infect Dis 1993, 17(6):968-75.

19. Borsuk $S$, et al: Identification of proteins from tuberculin purified protein derivative (PPD) by LC-MS/MS. Tuberculosis (Edinb) 2009, 89(6):423-30.

20. Harboe M: Antigens of PPD, old tuberculin, and autoclaved Mycobacterium bovis BCG studied by crossed immunoelectrophoresis. Am Rev Respir Dis 1981, 124(1):80-7.

21. Narasimhan SK, et al: Induced folding by chiral nonplanar aromatics. J Org Chem 2009, 74(18):7023-33.

22. Rupp ME, Schultz AW Jr, Davis JC: Discordance between tuberculin skin test results with two commercial purified protein derivative preparations. J Infect Dis 1994, 169(5):1174-5.

23. Lifson $A R$, et al: Discrepancies in tuberculin skin test results with two commercial products in a population of intravenous drug users. $J$ Infect Dis 1993, 168(4):1048-51.

24. Guld J, et al: Standardization of a new batch of purified tuberculin (PPD) intended for international use. Bull World Health Organ 1958, 19(5):845-951.

25. Ussetti $P$, et al: Tuberculin reactivity in the elderly. Comparison of PPDRT23 and PPD-CT68. Enferm Infecc Microbiol Clin 1996, 14(2):80-5.

26. Cho YS, et al: Deciphering the proteome of the in vivo diagnostic reagent "purified protein derivative" from Mycobacterium tuberculosis. Proteomics 2012, 12(7):979-91. 
27. Kuwabara S, Tsumita T: Letter: Primary structure of tuberculin-active protein from tubercule bacilli. Jpn J Exp Med 1974, 44(1):129-32.

28. Bardarov SS, et al: Characterization of PPD protein antigens in whole cell lysates of Mycobacterium bovis BCG. FEMS Microbio/ Lett 1990, 59(1-2):89-93.

29. Kitaura H, Kinomoto M, Yamada T: Ribosomal protein L7 included in tuberculin purified protein derivative (PPD) is a major heat-resistant protein inducing strong delayed-type hypersensitivity. Scand J Immunol 1999, 50(6):580-7.

30. Landi S: Preparation, Purification, and Stability of Tuberculin. Appl Microbiol 1963, 11:408-12.

31. Wong $\mathrm{HC}$, et al: The solution structure of acyl carrier protein from Mycobacterium tuberculosis. J Biol Chem 2002, 277(18):15874-80.

32. Elamin AA, et al: The Mycobacterium tuberculosis Ag85A is a novel diacylglycerol acyltransferase involved in lipid body formation. Mol Microbiol 2011, 81(6):1577-92.

33. Romero IC, et al: Identification of promoter-binding proteins of the fbp $\mathrm{A}$ and C genes in Mycobacterium tuberculosis. Tuberculosis (Edinb) 2010, 90(1):25-30.

34. Cobb AJ, Frothingham R: The GroES antigens of Mycobacterium avium and Mycobacterium paratuberculosis. Vet Microbiol 1999, 67(1):31-5.

35. Das Gupta T: B. Bandyopadhyay, and S.K. Das Gupta, Modulation of DNA-binding activity of Mycobacterium tuberculosis HspR by chaperones. Microbiology 2008, 154(Pt 2):484-90.

36. Rho BS, et al: Functional and structural characterization of a thiol peroxidase from Mycobacterium tuberculosis. J Mol Bio/ 2006, 361(5):850-63.

37. Gudkov AT: The L7/L12 ribosomal domain of the ribosome: structural and functional studies. FEBS Lett 1997, 407(3):253-6.

38. Reddy PV, et al: Iron storage proteins are essential for the survival and pathogenesis of Mycobacterium tuberculosis in THP-1 macrophages and the guinea pig model of infection. J Bacteriol 2012, 194(3):567-75.

39. Reddy MC, et al: Crystal structures of Mycobacterium tuberculosis Sadenosyl-L-homocysteine hydrolase in ternary complex with substrate and inhibitors. Protein Sci 2008, 17(12):2134-44

40. Hall G, et al: Structure of Mycobacterium tuberculosis thioredoxin C. Acta Crystallogr D: Biol Crystallogr 2006, 62(Pt 12):1453-7.

41. Tsai MH, Saier MH Jr: Phylogenetic characterization of the ubiquitous electron transfer flavoprotein families ETF-alpha and ETF-beta. Res Microbiol 1995, 146(5):397-404.

42. Hebert AM, et al: DNA polymorphisms in the pepA and PPE18 genes among clinical strains of Mycobacterium tuberculosis: implications for vaccine efficacy. Infect Immun 2007, 75(12):5798-805.

43. Lau Bonilla D, Dahl JL: The wag31 gene of Mycobacterium tuberculosis is positively regulated by the stringent response. FEMS Microbiol Lett 2011, 319(2):153-9.

44. Geisbrecht BV, et al: Design and optimization of a recombinant system for large-scale production of the MPT64 antigen from Mycobacterium tuberculosis. Protein Expr Purif 2006, 46(1):64-72.

45. Ragas A, et al: The Mycobacterium tuberculosis cell-surface glycoprotein apa as a potential adhesin to colonize target cells via the innate immune system pulmonary C-type lectin surfactant protein A. J Biol Chem 2007, 282(8):5133-42

46. Hovav $\mathrm{AH}$, et al: Aggravated infection in mice co-administered with Mycobacterium tuberculosis and the 27-kDa lipoprotein. Microbes Infect 2006, 8(7):1750-7.

47. Cole ST, et al: Deciphering the biology of Mycobacterium tuberculosis from the complete genome sequence. Nature 1998, 393(6685):537-44

48. Gu S, et al: Comprehensive proteomic profiling of the membrane constituents of a Mycobacterium tuberculosis strain. Mol Cell Proteomics 2003, 2(12):1284-96.

49. Wolfe LM, et al: Proteomic definition of the cell wall of Mycobacterium tuberculosis. J Proteome Res 2010, 9(11):5816-26.

50. Raman K, Yeturu K, Chandra N: targetTB: a target identification pipeline for Mycobacterium tuberculosis through an interactome, reactome and genome-scale structural analysis. BMC Syst Biol 2008, 2:109

51. Kunnath-Velayudhan S, et al: Dynamic antibody responses to the Mycobacterium tuberculosis proteome. Proc Natl Acad Sci USA 2010, 107(33):14703-8.

52. Fattorini $L$, et al: Recombinant GroES in combination with $\mathrm{CpG}$ oligodeoxynucleotides protects mice against Mycobacterium avium infection. J Med Microbio/ 2002, 51(12):1071-9.

53. Hussain $R$, et al: Immune profiling of leprosy and tuberculosis patients to 15-mer peptides of Mycobacterium leprae and M. tuberculosis GroES in a BCG vaccinated area: implications for development of vaccine and diagnostic reagents. Immunology 2004, 111(4):462-71.

54. Pais TF, et al: Analysis of T cells recruited during delayed-type hypersensitivity to purified protein derivative (PPD) versus challenge with tuberculosis infection. Immunology 1998, 95(1):69-75

55. Lemos JA, Giambiagi-Demarval M, Castro AC: Expression of heat-shock proteins in Streptococcus pyogenes and their immunoreactivity with sera from patients with streptococcal diseases. J Med Microbio/ 1998, 47(8):711-5.

56. Garufi G, Butler E, Missiakas D: ESAT-6-like protein secretion in Bacillus anthracis. J Bacteriol 2008, 190(21):7004-11.

57. Geluk A, et al: T-cell recognition of the HspX protein of Mycobacterium tuberculosis correlates with latent $M$. tuberculosis infection but not with M. bovis BCG vaccination. Infect Immun 2007, 75(6):2914-21.

58. Weldingh $\mathrm{K}$, et al: Assessing the serodiagnostic potential of 35 Mycobacterium tuberculosis proteins and identification of four novel serological antigens. J Clin Microbiol 2005, 43(1):57-65.

59. Bahk YY, et al: Antigens secreted from Mycobacterium tuberculosis: identification by proteomics approach and test for diagnostic marker. Proteomics 2004, 4(11):3299-307.

60. Khera $A$, et al: Elicitation of efficient, protective immune responses by using DNA vaccines against tuberculosis. Vaccine 2005, 23(48-49):5655-65.

61. Manca $C$, et al: Molecular cloning, purification, and serological characterization of MPT63, a novel antigen secreted by Mycobacterium tuberculosis. Infect Immun 1997, 65(1):16-23.

62. Elhay MJ, Oettinger T, Andersen P: Delayed-type hypersensitivity responses to ESAT-6 and MPT64 from Mycobacterium tuberculosis in the guinea pig. Infect Immun 1998, 66(7):3454-6.

63. He XY, et al: Comparative proteome analysis of culture supernatant proteins of Mycobacterium tuberculosis H37Rv and H37Ra. Microbes Infect 2003, 5(10):851-6.

64. Yang $\mathrm{H}$, et al: Three protein cocktails mediate delayed-type hypersensitivity responses indistinguishable from that elicited by purified protein derivative in the guinea pig model of Mycobacterium tuberculosis infection. Infect Immun 2011, 79(2):716-23.

65. Wu X, et al: Recombinant early secreted antigen target 6 protein as a skin test antigen for the specific detection of Mycobacterium tuberculosis infection. Clin Exp Immunol 2008, 152(1):81-7.

66. Arend SM, et al: Double-blind randomized Phase I study comparing rdESAT- 6 to tuberculin as skin test reagent in the diagnosis of tuberculosis infection. Tuberculosis (Edinb) 2008, 88(3):249-61.

67. Liu $C$, et al: Expression and purification of immunologically reactive DPPD, a recombinant Mycobacterium tuberculosis skin test antigen, using Mycobacterium smegmatis and Escherichia coli host cells. Can J Microbiol 2004, 50(2):97-105.

68. Pollock JM, et al: Specific delayed-type hypersensitivity responses to ESAT-6 identify tuberculosis-infected cattle. J Clin Microbiol 2003, 41(5):1856-60.

69. Campos-Neto A, et al: Evaluation of DPPD, a single recombinant Mycobacterium tuberculosis protein as an alternative antigen for the Mantoux test. Tuberculosis (Edinb) 2001, 81(5-6):353-8.

70. van Pinxteren $L A$, et al: Diagnosis of tuberculosis based on the two specific antigens ESAT-6 and CFP10. Clin Diagn Lab Immunol 2000, 7(2):155-60.

71. Colangeli R, et al: MTSA-10, the product of the Rv3874 gene of Mycobacterium tuberculosis, elicits tuberculosis-specific, delayed-type hypersensitivity in guinea pigs. Infect Immun 2000, 68(2):990-3

72. Oettinger T, et al: Mapping of the delayed-type hypersensitivity-inducing epitope of secreted protein MPT64 from Mycobacterium tuberculosis. Infect Immun 1995, 63(12):4613-8.

73. Vizcaino JA, et al: The Proteomics Identifications database: 2010 update. Nucleic Acids Res 2010, 38(Database issue):D736-42.

74. Harsha HC, Molina H, Pandey A: Quantitative proteomics using stable isotope labeling with amino acids in cell culture. Nat Protoc 2008, 3(3):505-16.

75. Rappsilber J, Mann M, Ishihama Y: Protocol for micro-purification, enrichment, pre-fractionation and storage of peptides for proteomics using StageTips. Nat Protoc 2007, 2(8):1896-906.

doi:10.1186/1559-0275-10-8

Cite this article as: Prasad et al:: Proteomic analysis of purified protein derivative of Mycobacterium tuberculosis. Clinical Proteomics 2013 10:8. 